\title{
On the Complexity of the Union of Fat Convex Objects in the Plane*
}

\author{
A. Efrat ${ }^{1}$ and M. Sharir ${ }^{1,2}$ \\ ${ }^{1}$ School of Mathematical Sciences, Tel Aviv University, \\ Ramat Aviv 69978, Israel \\ \{alone,sharir\}@math.tau.ac.il \\ ${ }^{2}$ Courant Institute of Mathematical Sciences, New York University, \\ New York, NY 10012, USA
}

\begin{abstract}
We prove a near-linear bound on the combinatorial complexity of the union of $n$ fat convex objects in the plane, each pair of whose boundaries cross at most a constant number of times.
\end{abstract}

\section{Introduction}

Let $\mathcal{C}$ be a collection of $n$ compact convex sets in the plane, satisfying the following properties:

(i) The objects in $\mathcal{C}$ are $\alpha$-fat, for some fixed $\alpha \geq 1$; that is, for each $c \in \mathcal{C}$ there exist two concentric disks $D \subseteq c \subseteq D^{\prime}$ such that the ratio between the radii of $D^{\prime}$ and $D$ is at most $\alpha$; see, e.g., [2].

(ii) For any pair of distinct objects $c, c^{\prime} \in \mathcal{C}$, their boundaries intersect in at most $s$ points, for some fixed constant $s$.

See [21] for more details concerning fat objects in the plane and in higher dimensions. Fat objects have recently been studied rather extensively, because in typical input scenes objects are very likely to be fat rather than long and skinny. The general goal of these studies is to design algorithms that are more efficient when their

* Work by Micha Sharir has been supported by the Hermann Minkowski - MINERVA Center for Geometry at Tel Aviv University, by a grant from the U.S.-Israeli Binational Science Foundation, by National Science Foundation Grants CCR-94-24398 and CCR-93-11127, and by a grant from the G.I.F.- the German Israeli Foundation for Scientific Research and Development. Work by Alon Efrat is part of his Ph.D. thesis, prepared under the supervision of Micha Sharir. Efrat's current address is: Department of Computer Science, Stanford University, Stanford, CA 94305, USA. 
input objects are fat, and this has been successful in many instances, including motion planning amidst fat obstacles [21], [22], binary space partition for fat objects [1], [4], visibility, hidden surface removal and depth orders [2], [14], range searching, point location, and data structures for similar kinds of queries [9], [13], [17], [18].

Our goal in this paper is to derive a near-linear upper bound on the combinatorial complexity of the union $U=\bigcup \mathcal{C}$, where we measure the complexity by the number of intersection points between the boundaries of the sets of $\mathcal{C}$ that lie on $\partial U$.

There are not too many results of this kind. If $\mathcal{C}$ is a collection of $\alpha$-fat triangles, ${ }^{1}$ then the complexity of $U$ is $O(n \log \log n)$ (with the constant of proportionality depending on $\alpha$ ) [16], and this bound improves to $O(n)$ if the triangles are nearly of the same size [2] or are infinite wedges [10]; see also [3]. See also [23] for additional results concerning fat polygons. If $\mathcal{C}$ is a collection of $n$ pseudodisks (arbitrary simply connected regions bounded by closed Jordan curves, each pair of whose boundaries intersect at most twice), then the complexity of $U$ is $O(n)$ [15]. Of course, without any additional conditions, the complexity of $U$ can be $\Omega\left(n^{2}\right)$, even for the case of (nonfat) triangles. Even for fat convex objects, something like condition (ii) must be assumed, or else the complexity of the union might be arbitrarily large.

The main result of this paper is

Theorem 1.1. The combinatorial complexity of the union of a collection $\mathcal{C}$ that satisfies conditions (i) and (ii) is $O\left(n^{1+c \sqrt{\log \log n / \log n}}\right)$, for some constant $c$ that depends on $\alpha$ and $s$.

Theorem 1.1 constitutes significant progress in the study of the union of planar objects, an area that has many algorithmic applications, such as finding the maximal depth in an arrangement of fat objects (see [12]), hidden surface removal in a collection of fat objects in 3-space [14], and point-enclosure queries in a collection of fat objects in the plane [13]. The proof of Theorem 1.1 is given in the following sections, and consists of the following stages. First, in Section 2, we review the notion of regular and irregular vertices, and recall the linear relationship between them due to Pach and Sharir [19]. Then, in Section 3, we introduce the machinery to be used in the proof. Informally, if our objects were fat convex polygons, then a nearlinear bound on the complexity of their union is known [16]. Consequently, we replace each object in $\mathcal{C}$ by an inscribed fat polygon, which leaves out a collection of convex "caps," whose properties are analyzed in detail. Finally, the proof of Theorem 1.1 itself is given in Section 4. The proof is somewhat involved and consists of several steps. An informal overview of the proof is given at the beginning of Section 4. The paper is concluded in Section 5 with a discussion of open problems related to our result.

\footnotetext{
${ }^{1}$ For triangles, there is an equivalent definition of fatness that requires all angles to be at least some fixed constant $\alpha_{0}$; in [16], this is called $\alpha_{0}$-fatness.
} 


\section{Regular and Irregular Vertices}

Let $\mathcal{C}$ be a collection of $n$ compact convex sets in the plane, and let $U$ denote their union. We assume that these regions are in general position, so that each pair of boundaries intersect in a finite number of points and properly cross at each point of intersection, and no three boundaries have a common point. (In this section we make no other assumptions on $\mathcal{C}$.) As already mentioned, we measure the combinatorial complexity of $U$ by the number of vertices of the arrangement $\mathcal{A}(\mathcal{C})$ of (the boundary curves of the objects in) $\mathcal{C}$ that lie on $\partial U$. (See [20] for details concerning arrangements; the vertices in question are points of intersection between pairs of boundaries of regions in $\mathcal{C}$.) We classify the arrangement vertices into two categories:

regular vertices: these are intersections between pairs of boundaries that intersect at only two points, and

irregular vertices: these are all the other boundary intersection points.

(In the preliminary version of the paper [11], we have referred to regular and irregular vertices as touching and shattering, respectively. The terms regular and irregular are taken from [19].)

Let $R(\mathcal{C})$ (resp. $I(\mathcal{C})$ ) denote the number of regular (resp. irregular) vertices of $U$. We use the following result of Pach and Sharir [19]:

\section{Theorem 2.1.}

$$
R(\mathcal{C}) \leq 2 I(\mathcal{C})+6 n-12
$$

for $n \geq 3$.

\section{Caps, Inscribed Fat Polygons, and Their Properties}

We now return to the case where $\mathcal{C}$ is a collection of compact convex sets satisfying conditions (i) and (ii) in the Introduction. Let $c \in \mathcal{C}$. We inscribe in $c$ a convex polygon $P_{c}$ defined as follows. We choose some constant integer parameter $t>12$, which also satisfies

$$
\frac{\alpha \sin (2 \pi / t)}{1-(\pi \alpha / t) \tan (\pi / t)}<1
$$

(clearly, this can always be enforced if $t=\Omega(\alpha)$ is chosen sufficiently large), and define $\theta_{j}=2 \pi j / t$, for $j=0,1, \ldots, t-1$. For each $j$, let $w_{j}=w_{j}(c)$ denote the (unique) point on $\partial c$ that has a tangent (that is, a supporting line) at orientation $\theta_{j}$ (tangents are assumed to be oriented so that $c$ lies to their left). We also define $w_{j}^{\prime}$, for $j=1, \ldots, t-1$, to be the point on $\partial c$ such that the length of the portion of $\partial c$ extending counterclockwise from $w_{0}$ to $w_{j}^{\prime}$ is $j / t$ times the perimeter of $c . P_{c}$ is defined to be the convex polygon whose vertices are $w_{0}, \ldots, w_{t-1}, w_{1}^{\prime}, \ldots, w_{t-1}^{\prime}$. (Note that $P_{c}$ may have fewer than $2 t-1$ vertices; this will be the case when some point $w_{i}$ accidentally coincides with some point $w_{j}^{\prime}$, or when $\partial c$ contains nonsmooth points whose tangent orientations span a sufficiently large interval, in which two or more points $w_{i}$ will coincide.) The difference $c \backslash P_{c}$ is the 


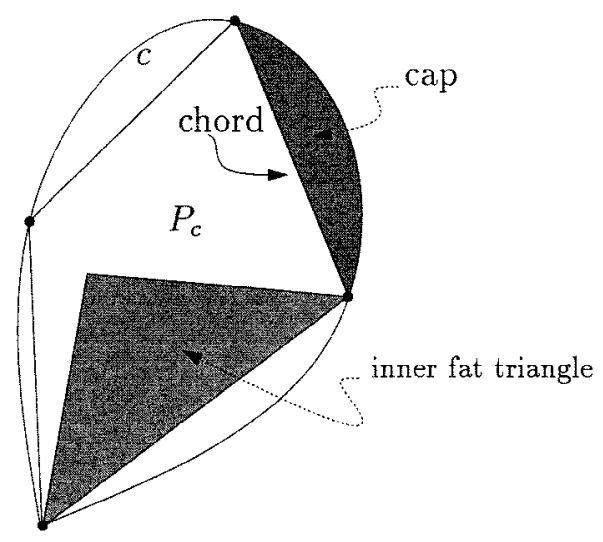

Fig. 1. The inscribed polygon $P_{c}$ and the corresponding caps; one inner fat triangle is also illustrated.

union of at most $2 t-1$ caps of $c$, where a cap is an intersection of $c$ with a halfplane. The chord of a cap is the intersection of $c$ with the line bounding the corresponding halfplane. An illustration of such an inscribed polygon and of the corresponding caps is shown in Fig. 1.

Lemma 3.1. The polygons $P_{c}$ are $\alpha^{\prime}$-fat, for

$$
\alpha^{\prime}=\frac{\alpha}{1-(\pi \alpha / t) \tan (\pi / t)}
$$

Proof. Since $c$ is $\alpha$-fat, there exist two concentric disks $D_{1} \subseteq c \subseteq D_{2}$, with respective radii $r_{1}, r_{2}$, such that $r_{2} \leq \alpha r_{1}$. Clearly, $P_{c} \subseteq D_{2}$. Let $K$ be one of the caps that constitute $c \backslash P_{c}$, and assume that $D_{1}$ intersects the chord $p q$ of $K$. It must do so at two points, or else its interior would have contained $p$ or $q$, contradicting the assumption that $D_{1} \subseteq c$. By definition, there exist two tangents to $c, \tau_{p}$ at $p$ and $\tau_{q}$ at $q$, whose orientations differ by at most $2 \pi / t$, and the distance $p q$ is smaller than $\sigma / t$, where $\sigma$ is the perimeter of $c$. Let $d$ denote the distance from the center $O$ of $D_{1}$ to $p q$. It is easy to see that $r_{1}-d$ is at most the height to $p q$ in the triangle bounded by $p q, \tau_{p}$ and $\tau_{q}$ (see Fig. 2), and a simple exercise shows that this height is at most $(p q / 2) \tan (\pi / t)$. Hence

$$
r_{1}-d \leq \frac{p q}{2} \tan \frac{\pi}{t}<\frac{\sigma}{2 t} \tan \frac{\pi}{t} \leq \frac{2 \pi \alpha r_{1}}{2 t} \tan \frac{\pi}{t}=\frac{\pi \alpha r_{1}}{t} \tan \frac{\pi}{t}
$$

where the last inequality follows from the fact that $c \subseteq D_{2}$. Hence

$$
d \geq r_{1}\left(1-\frac{\pi \alpha}{t} \tan \frac{\pi}{t}\right)
$$

This implies that the disk concentric with $D_{1}$ and having radius $r_{1}(1-(\pi \alpha / t) \tan (\pi / t))$ is contained in $P_{c}$, and this completes the proof of the lemma. 


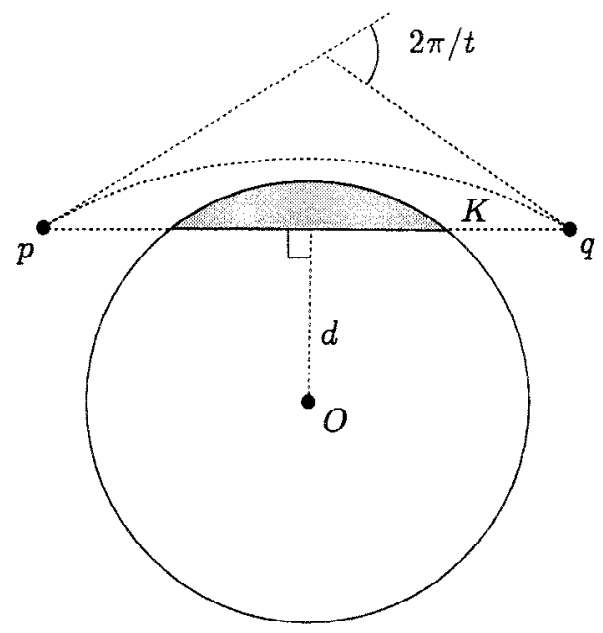

Fig. 2. The proof of Lemma 3.1.

Let $c \in \mathcal{C}$, and let $O$ denote the common center of two disks $D_{1} \subseteq P_{c} \subseteq D_{2}$, such that their respective radii $r_{1}, r_{2}$ satisfy $r_{2} \leq \alpha^{\prime} r_{1}$. Let $p q$ be an edge of $P_{c}$. The convexity of $P_{c}$ and the fact that $D_{1} \subseteq P_{c}$ are easily seen to imply that the angle $O p q$ must be at least the angle $\beta$ between $O p$ and the tangent to $D_{1}$ from $p$, which satisfies $\sin \beta=r_{1} /|O p| \geq r_{1} / r_{2} \geq 1 / \alpha^{\prime}$. Similarly, the angle $O q p$ must also be at least $\arcsin \left(1 / \alpha^{\prime}\right)$. It follows that we can find a point $v$ inside $O p q$, such that all the angles of the triangle $v p q$ are at least

$$
\beta_{0}=\min \left\{\arcsin \left(\frac{1}{\alpha^{\prime}}\right), \frac{\pi}{3}\right\} .
$$

Note that, by the choice of $t$ in (1), $\beta_{0}>2 \pi / t$.

We repeat this analysis to each edge of each polygon, and replace the polygons $P_{c}$ by the collection of resulting triangles $v p q$. We refer to these triangles as inner fat triangles. Let $\mathcal{T}=\mathcal{T}(\mathcal{C})$ denote the collection of inner fat triangles. Clearly, $|\mathcal{T}| \leq(2 t-1) n$. As an immediate consequence of [16], we have:

Lemma 3.2. The union $U_{\mathcal{T}}$ of the triangles in $\mathcal{T}$ has $O(n \log \log n)$ vertices.

Note that the union $U_{\mathcal{T}}$ does not necessarily cover the whole interior of a polygon $P_{c}$. Nevertheless, it covers the portion of any such polygon that lies near its boundary.

Let $v$ be an irregular vertex of $\partial U$, incident to two sets $a, b \in \mathcal{C}$. Let $K_{a}, K_{b}$ be the respective caps of $a, b$ that contain $v$, and let $p_{a} q_{a}, p_{b} q_{b}$ denote their respective chords. Consider the convex set $R=K_{a} \cap K_{b}$.

Lemma 3.3. At least one of the chords $p_{a} q_{a}, p_{b} q_{b}$ meets $\partial R$.

Proof. Indeed, suppose to the contrary that both chords are disjoint from $R$. It follows that $R=a \cap b$, and that $\partial R$ contains at least four points of intersection between $\partial a$ and 


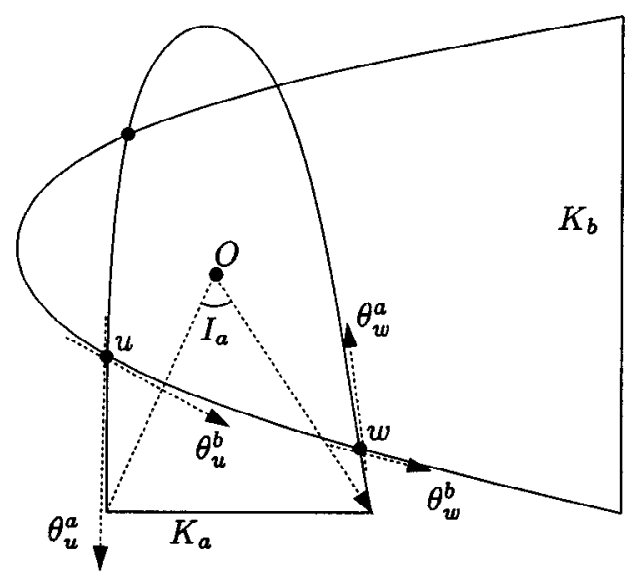

Fig. 3. Two intersecting caps without a chordal intersection.

$\partial b$. Moreover, let $O$ be an interior point of $R$, and consider $\partial K_{a}$ and $\partial K_{b}$ as graphs of two respective functions $r=K_{a}(\theta), r=K_{b}(\theta)$, in polar coordinates about $O$. Note that $\partial R$ is the graph of the pointwise minimum of $K_{a}$ and $K_{b}$. There is an angular interval $I_{a}$ over which $K_{a}(\theta)$ is attained at the chord of $K_{a}$, and a similar interval $I_{b}$ for the chord of $K_{b}$. These intervals must be disjoint, or else $\partial R$ would overlap at least one of these chords, contrary to assumption. See Fig. 3.

Let $u$ (resp. $w$ ) denote the first vertex of $\partial R$ that we encounter as we rotate about $O$ clockwise (resp. counterclockwise) from $I_{a}$ (clearly, no vertex of $\partial R$ has an orientation in $I_{a}$ ). In the angular interval that runs counterclockwise from $u$ to $w$, the boundary of $R$ is attained by $\partial b$. Moreover, as we traverse, in counterclockwise direction, the portion of $\partial b$ that lies on $\partial K_{b}$, we first encounter $u$ and then $w$, and the reverse order is obtained along $\partial a$. See Fig. 3.

Let $\theta_{u}^{a}, \theta_{w}^{a}$ denote the orientations of the tangents to $a$ at $u$ and $w$, respectively, and let $\theta_{u}^{b}, \theta_{w}^{b}$ denote the corresponding tangent orientations for $b$. (If any of these tangents is not unique, we fix an arbitrary tangent among those that are available.) The circular counterclockwise order of these four orientations is $\left(\theta_{u}^{a}, \theta_{u}^{b}, \theta_{w}^{b}, \theta_{w}^{a}\right)$, and they partition the circular range of orientations into four angular intervals that we denote by $\left(\theta_{u}^{a}, \theta_{u}^{b}\right)$, $\left(\theta_{u}^{b}, \theta_{w}^{b}\right),\left(\theta_{w}^{b}, \theta_{w}^{a}\right)$, and $\left(\theta_{w}^{a}, \theta_{u}^{a}\right)$. Each of the second and fourth intervals has length at most $2 \pi / t$ (since the endpoints of any of these intervals are two tangent orientations within a single cap), and each of the first and third intervals has length at most $\pi$ (the total amount by which the tangent to the convex set $R$ can turn at a fixed point of its boundary is at most $\pi$ ). It follows that each of the lengths of the first and third intervals is at least $\pi-4 \pi / t>2 \pi / 3$.

We now repeat the whole analysis in the last two paragraphs by interchanging $a$ and $b$. This yields two vertices $u^{\prime}, w^{\prime}$ of $\partial R$, such that the turning angle of the tangents to $R$ at each of these vertices is also greater than $2 \pi / 3$. It is easily verified that among the vertices $u, w, u^{\prime}, w^{\prime}$ there exist at least three distinct vertices, or else $\partial a$ and $\partial b$ would have intersected at only two points, contrary to assumption. We have thus obtained at 


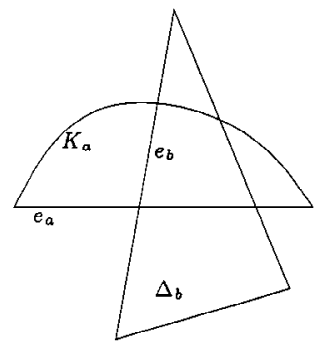

(i)

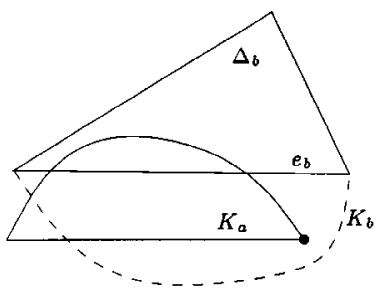

(iv.a)

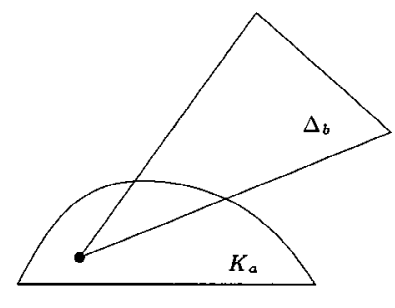

(ii)

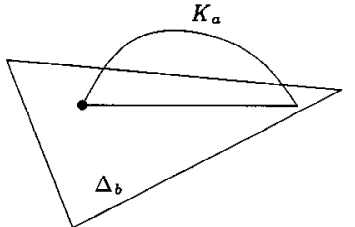

(iii)

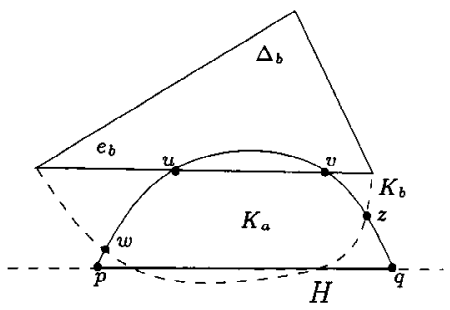

(iv.b)

Fig. 4. Illustrating the various cases in Lemma 3.4.

least three vertices of $R$ such that the turning angle of the tangents at each of them is greater than $2 \pi / 3$, which is impossible, because the overall turning angle for a convex set is $2 \pi$. This contradiction completes the proof of the lemma.

Lemma 3.4. Let $K_{a}$ be a cap of some set $a \in \mathcal{C}$, with chord $e_{a}$, and let $\Delta_{b}$ be an inner fat triangle in $\mathcal{T}$, obtained from the polygon $P_{b}$, for some $b \in \mathcal{C}$, such that the chord $e_{b}$ of $\Delta_{b}$ (i.e., the side of $\Delta_{b}$ that is also a chord of an adjacent cap of $b$ ) crosses $\partial K_{a}$. Then one of the following cases must occur:

(i) $e_{a}$ crosses $\partial \Delta_{b}$ (as in Fig. 4(i)).

(ii) $K_{a}$ contains a vertex of $\Delta_{b}$ that is an endpoint of $e_{b}$ (as in Fig. 4(ii)).

(iii) $\Delta_{b}$ contains a vertex of $K_{a}$ (as in Fig. 4(iii)).

(iv) $\partial K_{a}$ and $\partial \Delta_{b}$ cross exactly twice, at two points that lie on $\partial a$ and on $e_{b}$, and $e_{a}$ is disjoint from $K_{a} \cap \Delta_{b}$. Furthermore, let $K_{b}$ denote the cap of $b$ that shares the same chord $e_{b}$ with $\Delta_{b}$. Then either $K_{b}$ contains an endpoint of $e_{a}$ (as in Fig. 4(iv.a)), or $\partial a$ and $\partial b$ intersect only twice (as in Fig. 4(iv.b)).

Proof. Suppose that cases (i) and (ii) do not occur. That is, $e_{a}$ does not cross $\partial \Delta_{b}$ and no vertex of $\Delta_{b}$ that is an endpoint of $e_{b}$ lies in $K_{a}$. Then $e_{b}$ must intersect $\partial K_{a}$ at two points, $u, v$, both lying on $\partial a$. Therefore $e_{b}$ splits $K_{a}$ into two subregions, the region $K_{a}^{\prime}$ that contains $e_{a}$, and the complementary region $K_{a}^{\prime \prime}$. Denote the range of the orientations 


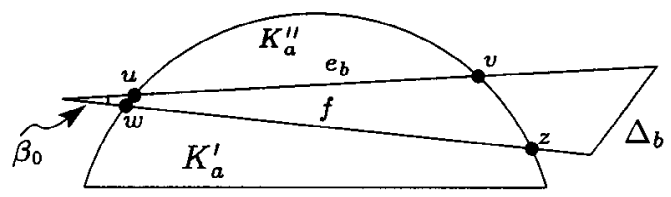

(a)

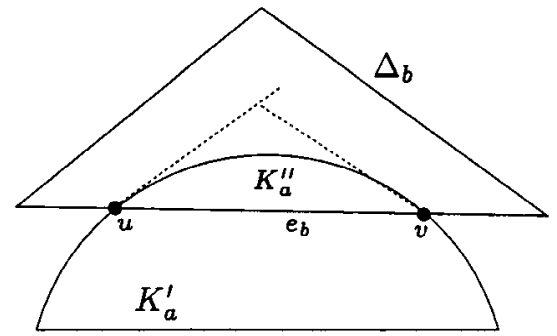

(b)

Fig. 5. Two patterns of intersection of a cap $K_{a}$ and an inner fat triangle $\Delta_{b}$.

of the tangents to $a$ at the points of $K_{a}$ by $\left(\theta_{0}, \theta_{0}^{\prime}\right)$, where $\theta_{0}<\theta_{0}^{\prime} \leq \theta_{0}+2 \pi / t$. Clearly, the orientations of $e_{a}$ and of $e_{b}$ also lie in this range. Two cases can arise:

Case (1): $\Delta_{b}$ overlaps $K_{a}^{\prime}$ and is disjoint from $K_{a}^{\prime \prime}$ (see Fig. 5(a)). If $K_{a}^{\prime}$ is fully contained in $\Delta_{b}$, then $u$ and $v$ are the only two points of intersection between $\partial K_{a}$ and $\partial \Delta_{b}$, and, moreover, $\Delta_{b}$ contains both vertices of $K_{a}$, so we are in case (iii). Otherwise, since, by assumption, $\Delta_{b}$ does not intersect $e_{a}$ and does not have a vertex inside $K_{a}^{\prime}$, one of its other edges, $f$, must also cross $\partial K_{a}$ twice, at two points $w, z$, lying on $\partial a$, so that the four points $w, u, v, z$ appear in this order along $\partial K_{a}$. In this case the orientation of $f$ also lies in the range $\left(\theta_{0}, \theta_{0}^{\prime}\right)$, and thus the angle between $e$ and $f$, which is $\geq \beta_{0}$, is at most $2 \pi / t$, a contradiction.

Case (2): $\Delta_{b}$ overlaps $K_{a}^{\prime \prime}$ and is disjoint from $K_{a}^{\prime}$ (see Fig. 5(b)). We claim that in this case $\Delta_{b}$ fully contains $K_{a}^{\prime \prime}$, so $u$ and $v$ are the only two intersection points of $\partial K_{a}$ and $\partial \Delta_{b}$. Since the orientations of $e_{b}$ and of the tangents (or, rather, any tangents) to $a$ at $u$ and at $v$ all lie in the range $\left(\theta_{0}, \theta_{0}^{\prime}\right)$, it follows that the angles between $e$ and these tangents are both at most $2 \pi / t$. However, the angles of $\Delta_{b}$ at the endpoints of $e$ are both $\geq \beta_{0}$, and are therefore larger. It follows that the triangle bounded by $e$ and by two such tangents is fully contained in $\Delta_{b}$, from which the claim follows readily.

Finally, suppose that $K_{b}$ does not contain any of the endpoints $e_{a}$. Let $p$ and $q$ be the endpoints of $e_{a}$, so that $p, u, v, q$ appear in this order along $\partial a$. Then the portion of $\partial K_{b}$ along $\partial b$ must cross the portion of $\partial K_{a}$ along $\partial a$ at least twice, at one point $w$ between $p$ and $u$ and at another point $z$ between $v$ and $q$ (see Fig. 4(iv.b)). We claim that $w$ and $z$ are the only two intersection points of $\partial a$ and $\partial b$. Indeed, suppose, with no loss of generality, that $e_{a}$ lies along the $x$-axis and that $K_{a}$ lies above it. Then $\gamma_{a} \equiv \partial a \cap K_{a}$ is a downward-concave $x$-monotone arc. Moreover, the absolute value of the orientation of $e_{b}$ is at most $2 \pi / t$, so the orientation of any tangent to $\gamma_{b} \equiv \partial b \cap K_{b}$ has absolute value $\leq 4 \pi / t$, which is easily seen to imply that $\gamma_{b}$ is also $x$-monotone and downward-convex. It follows that $\gamma_{a}$ and $\gamma_{b}$ cross each other exactly twice (at $w$ and $z$ ). We claim that there 
can be no other point of intersection between $\partial a$ and $\partial b$. Indeed, any such point must lie either in the halfplane below $e_{a}$ or in the halfplane above $e_{b}$. Consider the halfplane $H$ lying below $e_{a}$ (the second case, where we consider the halfplane above $b$, is treated in a fully symmetric manner). It is easy to see that any such intersection must lie on $\gamma_{b}$. However, if $\gamma_{b}$ reaches $H$ it must cross $e_{a}$ twice. Arguing as above, it follows that the portion of $\gamma_{b}$ in $H$ is fully contained in the inner fat triangle of $P_{a}$ that has $e_{a}$ as a chord, and hence it cannot intersect $\partial a$ at all. This shows that condition (iv) holds, and thus completes the proof of the lemma. (Note that these arguments also imply that, in any configuration of case (iv), $\partial a \cap K_{a}$ and $\partial b \cap K_{b}$ can intersect in at most two points; they intersect in one or zero points if and only if $K_{b}$ contains an endpoint of $e_{a}$.)

\section{The Analysis of the Complexity of the Union}

The proof proceeds by induction on $n$, keeping $\alpha$ and $s$ fixed. Let $F(n)$ denote the maximum number of vertices of the union of any collection $\mathcal{C}$ of $n$ compact convex objects, satisfying conditions (i) and (ii) of the Introduction. We will show that $F(n) \leq$ $n^{1+c \sqrt{\log \log n / \log n}}$, where $c$ is a sufficiently large constant, depending on $\alpha$ and $s$. By choosing $c$ sufficiently large, this will hold for all $n \leq n_{0}$, where the value of $n_{0}$ will be defined below. Suppose then that $n>n_{0}$ and that the claim holds for all $n^{\prime}<n$.

Overview of the Proof. The proof proceeds through several reduction steps, each making the problem more restricted. In the first step we exploit the fatness of the objects to show that the plane can be covered by a system of axis-parallel rectangles such that each vertex $v$ of the union can be assigned to a rectangle $R$ that contains it so that at least one of the two sets whose boundaries contain $v$ is relatively large with respect to $R$, and hence can be stabbed by at least one of $O(1)$ points scattered around $R$. This is important, because the boundary of the union of a collection $\mathcal{C}_{1}$ of convex sets that all contain a fixed point $O$ can be regarded as the upper envelope of the individual boundaries, in polar coordinates around $O$, and thus has near-linear complexity. We have thus reduced the problem into that of bounding the number of "mixed" vertices of the union of two collections $\mathcal{C}_{1}, \mathcal{C}_{2}$ of fat convex objects (vertices lying on the boundaries of an object in $\mathcal{C}_{1}$ and of an object in $\mathcal{C}_{2}$ ), so that all objects in $\mathcal{C}_{1}$ contain a fixed point $O$.

In the second step we replace each object of $\mathcal{C}_{2}$ by its inner inscribed fat polygon, and bound the complexity of $U^{*}=U_{1} \cup \tilde{U}_{2}$, where $U_{1}$ is the union of $\mathcal{C}_{1}$ and $\tilde{U}_{2}$ is the union of the fat inner polygons of the objects in $\mathcal{C}_{2}$. Each of the unions $U_{1}$ and $\tilde{U}_{2}$ has near-linear complexity, so it suffices to bound the number of "mixed" vertices of the union. By passing rays from $O$ through each vertex of $U_{1}$, we partition the plane into a near-linear number of wedges, and we bound the number of mixed vertices of $U^{*}$ within each slice separately, using several charging schemes, where the mixed vertices are charged to vertices of $U_{1}$ or of $\tilde{U}_{2}$, leading to a near-linear bound on the number of these vertices.

Finally, in the third step we bound the number of mixed vertices of the union of $\mathcal{C}_{1} \cup \mathcal{C}_{2}$. The analysis here is somewhat more involved. Several kinds of vertices can be charged to vertices of unions analyzed in the preceding steps. Other kinds of vertices are charged to intersections of boundaries that lie at shallow depth, meaning that they are 
not vertices of the union but are contained in only a small number of objects. Using the Clarkson-Shor analysis technique, this leads to a recurrence formula for $F(n)$, whose solution yields the desired bound.

Step I. For each $c \in \mathcal{C}$, let $Q_{c}$ denote a smallest axis-parallel square enclosing $c$. We construct a two-dimensional hereditary segment tree on the collection $\mathcal{Q}=\left\{Q_{c} \mid c \in \mathcal{C}\right\}$ as follows. We construct a one-dimensional segment tree $T_{1}$ on the $x$-projections of the squares in $\mathcal{Q}$. We make $T_{1}$ hereditary, as in [5], by propagating a square $Q_{c}$ that is normally stored at some node $\xi$ of $T_{1}$ to all ancestors of $\xi$. In this manner, each node $\xi$ of $T_{1}$ stores two lists: the standard list $L_{1}(\xi)$ of squares stored at $\xi$ (we refer to these squares as long), and a list $S_{1}(\xi)$ of squares that were propagated to $\xi$ from its (proper) descendants (we refer to these squares as short). The total length of all these lists is still $O(n \log n)$.

We now take each node $\xi$ of $T_{1}$, and construct a secondary hereditary segment tree $T_{2}^{(\xi)}$ on the $y$-projections of the squares in $L_{1}(\xi) \cup S_{1}(\xi)$. Again, each node $\eta$ of any secondary tree stores two lists: the standard list $L_{2}(\eta)$ of "long" squares, and a list $S_{2}(\eta)$ of "short" squares, propagated from the proper descendants of $\eta$. The total size of the structure is $O\left(n \log ^{2} n\right)$.

Let $v$ be a vertex of the union, lying on the boundaries of two sets $a, b \in \mathcal{C}$. We take the leaf $\zeta$ of $T_{1}$ whose $x$-interval contains the $x$-coordinate of $v$, and consider the path from $\zeta$ to the root of $T_{1}$. There is a unique node $\xi$ on that path such that one of $Q_{a}, Q_{b}$ is stored at $L_{1}(\xi)$ and the other square is stored at $L_{1}(\xi) \cup S_{1}(\xi)$. Repeating this for the secondary tree $T_{2}^{(\xi)}$ and the $y$-coordinate, we obtain a unique node $\eta$ of $T_{2}^{(\xi)}$ (in fact of the whole structure) such that $v$ lies in the rectangle $R_{\eta}$, defined as the cartesian product of the $x$-interval associated with $\xi$ and the $y$-interval associated with $\eta$, and such that one of the squares $Q_{a}, Q_{b}$ is stored at $L_{2}(\eta)$ and the other is stored at $L_{2}(\eta) \cup S_{2}(\eta)$.

Our strategy is thus to iterate over all vertices $\eta$ of all the secondary trees $T_{2}^{(\xi)}$, and, for each fixed $\xi, \eta$, prove a near-linear upper bound on the number of vertices $v$ of $U$, incident to a pair of objects $a, b \in \mathcal{C}$, satisfying

- $v \in R_{\eta}$;

- both squares $Q_{a}, Q_{b}$ are in $L_{2}(\eta) \cup S_{2}(\eta)$ (and thus also in $L_{1}(\xi) \cup S_{1}(\xi)$ ); and

- at least one of these squares is in $L_{1}(\xi)$, and at least one is in $L_{2}(\eta)$.

We prune the sets $L_{2}(\eta), S_{2}(\eta)$, so as to retain only squares $Q_{a}$ whose object $a$ intersects $R_{\eta}$. Clearly, the $a$ and $b$ above are not pruned by this rule. We continue to use the same notation $L_{2}(\eta), S_{2}(\eta)$ for the pruned sets.

Let $\xi$ and $\eta$ be fixed.

\section{Lemma 4.1.}

(a) Suppose that the height of $R_{\eta}$ is larger than or equal to its width. Then there exists a set $P_{\eta}$ of $O(1)$ points, all lying in the rectangle $R_{\eta}^{\prime}$ obtained by scaling up $R_{\eta}$ by a factor of 2 about its center, such that any $a \in \mathcal{C}$ with $Q_{a} \in L_{2}(\eta)$ has a nonempty intersection with $P_{\eta}$.

(b) Suppose that the height of $R_{\eta}$ is smaller than its width. Then there exists a set $P_{\eta}$ of $O(1)$ points, as above, such that any $a \in \mathcal{C}$ with $Q_{a} \in L_{1}(\xi) \cap\left(L_{2}(\eta) \cup S_{2}(\eta)\right)$ has a nonempty intersection with $P_{\eta}$. 
Proof. Consider the proof of (a). Let $a \in \mathcal{C}$ be such that $Q_{a} \in L_{2}(\eta)$. Since (i) $a$ intersects $R_{\eta}$, (ii) the $y$-projection of $Q_{a}$ contains that of $R_{\eta}$, (iii) the $y$-projection of $R_{\eta}^{\prime}$ is at least as large as its $x$-projection, and (iv) $a$ is $\alpha$-fat, it follows that the area of $a \cap R_{\eta}^{\prime}$ is at least some fixed portion of the area of $R_{\eta}^{\prime}$ (see [13]). Hence, if we place a sufficiently dense grid of $O(1)$ points within $R_{\eta}^{\prime}$, at least one of them will lie in $a$. The proof of (b) is fully symmetric.

Let $P_{\eta}$ be the point set yielded by Lemma 4.1, and fix a point $p$ in $P_{\eta}$. Let $\mathcal{C}_{1}(p)$ (resp. $\left.\mathcal{C}_{2}(p)\right)$ denote the collection of sets $a \in \mathcal{C}$ that contain (resp. do not contain) $p$, and whose enclosing squares $Q_{a}$ are in $L_{2}(\eta) \cup S_{2}(\eta)$. The preceding analysis implies that each vertex $v$ of $U$ that satisfies the above conditions has at least one $p \in P_{\eta}$ such that one of the sets whose boundaries contain $v$ lies in $\mathcal{C}_{1}(p)$ and the other set lies in $\mathcal{C}_{1}(p) \cup \mathcal{C}_{2}(p)$.

All these reductions imply that it suffices to solve the following problem: We are given two families $\mathcal{C}_{1}, \mathcal{C}_{2}$ of $\alpha$-fat convex objects in the plane, each pair of whose boundaries intersect at most $s$ times. We are also given that all the objects in $\mathcal{C}_{1}$ contain a fixed point, which from now on we take to be the origin. We want to obtain a near-linear upper bound for the number of vertices of the union of $\mathcal{C}_{1} \cup \mathcal{C}_{2}$ that lie on the boundary of at least one set in $\mathcal{C}_{1}$.

Once this has been accomplished, we sum these bounds, for the sets $\mathcal{C}_{1}(p), \mathcal{C}_{2}(p)$, over the $O(1)$ points $p \in P_{\eta}$, and over all nodes $\xi \in T_{1}$ and $\eta \in T_{2}^{(\xi)}$, to obtain an overall near-linear bound. The exact details are somewhat more complex, because they involve the derivation of a recurrence formula for $F(n)$; see toward the end of the proof.

Put $m_{1}=\left|\mathcal{C}_{1}\right|$ and $m_{2}=\left|\mathcal{C}_{2}\right|$. Let $U_{1}$ denote the union of $\mathcal{C}_{1}$. If we represent the boundary of every $c \in \mathcal{C}_{1}$ as the graph of a function $r=r_{c}(\theta)$ in polar coordinates, the boundary of $U_{1}$ is the graph of the upper envelope of these functions. Since any pair of these functions intersect in at most $s$ points, the number of vertices of $U_{1}$ is at most $\lambda_{s}\left(m_{1}\right)[20]$.

For each $a \in \mathcal{C}_{2}$, we construct an inner fat inscribed polygon $P_{a}$, as in Section 3, with the additional proviso that the vertices of $P_{a}$ also include the two points on $\partial a$ that have extreme clockwise and counterclockwise orientations about the origin. (Since $a$ does not contain the origin, these points are well defined, except that any of them may actually be replaced by a radially directed segment on $\partial a$; in this case, the two endpoints of such an interval are assumed to be vertices of $P_{a}$.)

Let $\tilde{U}_{2}$ denote the union of the polygons $P_{a}$, for $a \in \mathcal{C}_{2}$. Note that any vertex of $\tilde{U}_{2}$ is also a vertex of the union of the inscribed fat triangles of the polygons $P_{a}$. By the result of [16] (see also Lemma 3.2), the number of vertices of $\tilde{U}_{2}$ is thus $O\left(m_{2} \log \log m_{2}\right)$.

Step II. As an intermediate step, we bound the complexity of $U^{*}=U_{1} \cup \tilde{U}_{2}$. It suffices to bound the number of "mixed" vertices of $U^{*}$, that is, vertices that lie on both $\partial U_{1}$ and $\partial \tilde{U}_{2}$. To begin with, we take each $a \in \mathcal{C}_{1}$ and modify its inner fat inscribed polygon $P_{a}$ by adding all the vertices of $\partial U_{1}$ that lie on $\partial a$ as vertices of $P_{a}$. We obtain a collection of new polygons $P_{a}^{*}$, such that any one of them may now have more than a constant number of vertices. Still, the overall number of their vertices is $O\left(\lambda_{s}\left(m_{1}\right)\right)$. Note that the new polygons $P_{a}^{*}$ are at least as fat as the original polygons. 


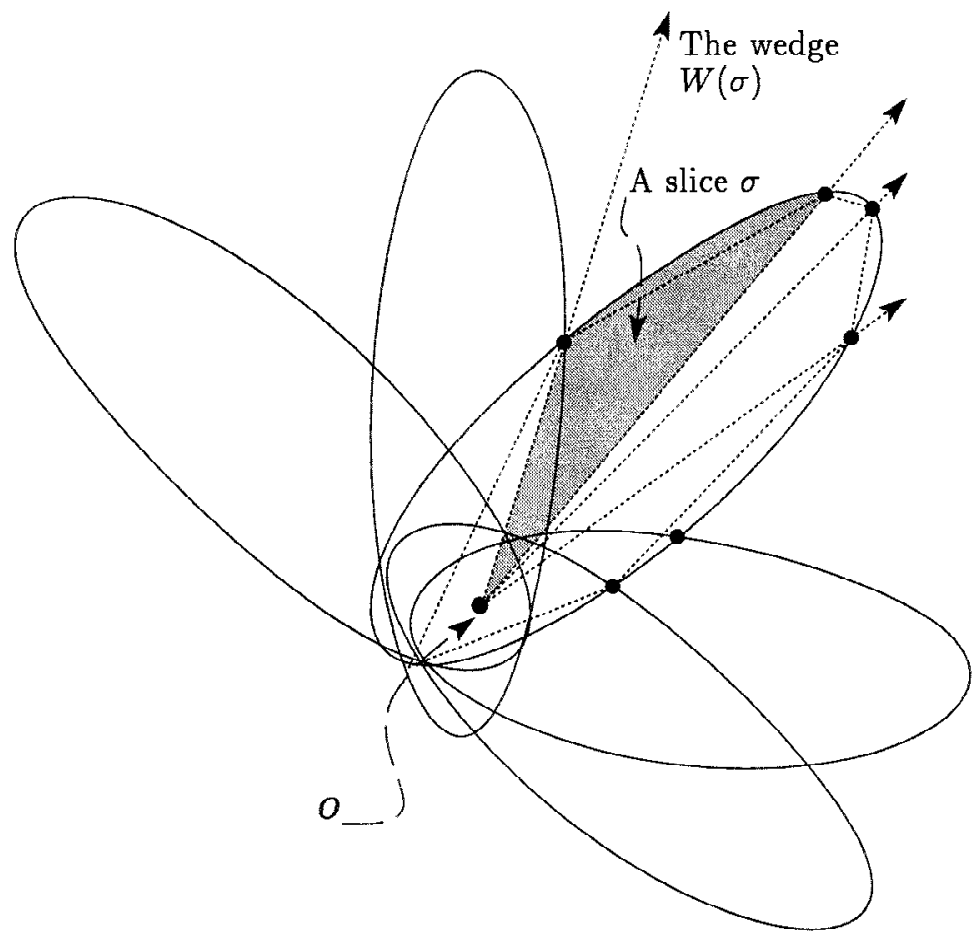

Fig. 6. The union $U_{1}$ of $\mathcal{C}_{1}$ and its decomposition into slices. A slice $\sigma$ and its containing wedge $W(\sigma)$ are shown.

Next, we take each point $w$ which is either a vertex of $U_{1}$ or a vertex of some $P_{a}$, for $a \in \mathcal{C}_{1}$, and connect it by a straight segment to the origin. These segments partition $U_{1}$ into "slices," each bounded by two of these segments and by a portion of the boundary of a single set in $\mathcal{C}_{1}$. Each slice is further subdivided, by the chord connecting its two vertices, into a cap (as in Section 3) and a triangle with the origin as a vertex. We also define the wedge $W(\sigma)$ of a slice $\sigma$ to be the positive hull of $\sigma$ (it is the wedge with the origin as apex and with two bounding rays containing the segments bounding $\sigma$ ). See Fig. 6 for an illustration.

Let $v$ be a mixed vertex, that is, a vertex lying on the boundary of a set $a \in \mathcal{C}_{1}$ and on the boundary of a polygon $P_{b}$ for some $b \in \mathcal{C}_{2}$. Let $\sigma$ be the slice of $U_{1}$ that contains $v$, and let $e$ be the edge of $P_{b}$ that contains $v$. Suppose first that $P_{b}$ and the origin lie on opposite sides of the line containing $e$. If $e$ intersects $\sigma \cap \partial a$ at two points, then Lemma 3.4(iv) implies that $P_{b}$ (or, more precisely, the inner fat triangle of $P_{b}$ having $e$ as an edge) and $\sigma$ intersect in just two points (one of which is $v$ ), so $v$ is a regular vertex of $U^{*}$ (viewed as the union of the slices and the inscribed fat polygons of the sets in $\left.\mathcal{C}_{2}\right)$. By Theorem 2.1, the number of such vertices is at most $2 I+6\left(m_{1}+m_{2}\right)-12$, where $I$ is the number of irregular vertices of $U^{*}$. It thus suffices to bound the number of irregular mixed vertices of $U^{*}$, so the above case can be ignored. If $e$ intersects $\sigma \cap \partial a$ only at $v$, then it must intersect the chord of $\sigma$, or end inside the cap. This means that if 
we follow $e$ from $v$ into $\sigma$, we encounter there a vertex of the union $S_{1} \cup \tilde{U}_{2}$, where $S_{1}$ is the star-shaped polygon composed of all the slice chords. We then charge $v$ to the first such vertex that we encounter, and note that this charging is unique.

Lemma 4.2. The complexity of $S_{1} \cup \tilde{U}_{2}$ is $O\left(\left(\lambda_{s}\left(m_{1}\right)+m_{2}\right) \log \log n\right)$.

Proof. We take the modified inscribed fat polygons $P_{a}^{*}$, for $a \in \mathcal{C}_{2}$, and replace each of them by their corresponding inner fat triangles, as in Section 3. It is clear that $\partial S_{1}$ is contained in the boundary of the union of these triangles. It follows that every vertex of $S_{1} \cup \tilde{U}_{2}$ is also a vertex of the union of $O\left(\lambda_{s}\left(m_{1}\right)+m_{2}\right) \alpha^{\prime}$-fat triangles, and the lemma thus follows from [16].

Hence, the number of mixed vertices $v$ in the preceding case, in which $P_{b}$ and the origin lie on opposite sides of the line containing $e$, is $O\left(\left(\lambda_{s}\left(m_{1}\right)+m_{2}\right) \log \log n\right)$.

We may therefore assume that $P_{b}$ and the origin lie on the same side of the line containing $e$. The intersection of $e$ and of $\sigma \cap \partial a$ consists of one or two points. Suppose first that there is only one point of intersection, namely $v$. We trace $e$ from $v$ into $\sigma$, and note that the line containing $e$ must intersect the boundary of the cap of $\sigma$ at another point $v^{\prime}$. If we encounter a vertex $w$ of $\tilde{U}_{2}$ (which can be the endpoint of $e$ or an earlier point on $e$ ) before reaching $v^{\prime}$, we charge $v$ to $w$ and note that $w$ can be charged at most twice, once along each of the two edges of $\partial \tilde{U}_{2}$ incident to it. The overall number of such vertices $v$ is $O\left(m_{2} \log \log m_{2}\right)$.

Otherwise we reach $v^{\prime}$, which, by assumption, necessarily lies on the chord of $\sigma$. As above, $v^{\prime}$ is a vertex of the union $S_{1} \cup \tilde{U}_{2}$, and we can charge $v$ to $v^{\prime}$, note that the charging is unique (it can occur only once, along the single edge of $\partial\left(S_{1} \cup \tilde{U}_{2}\right)$ that is incident to $v^{\prime}$ and lies on $\left.\partial \tilde{U}_{2}\right)$, and conclude, by Lemma 4.2, that the number of vertices $v$ in this subcase is $O\left(\left(\lambda_{s}\left(m_{1}\right)+m_{2}\right) \log \log n\right)$.

Suppose then that $e$ intersects $\sigma \cap \partial a$ at two points, $v$ and $v^{\prime}$, and that its portion within $\sigma$ contains no vertex of $\tilde{U}_{2}$ (otherwise we can charge $v$ to the first such vertex along $e$, as above). Hence $v^{\prime}$ is also a vertex of $U^{*}$. If $e$ terminates within the wedge $W(\sigma)$ of $\sigma$, we can charge $v$ and $v^{\prime}$ to such an endpoint. This charging is "almost unique": Since these wedges are pairwise openly disjoint, the endpoint determines $e$ (there are in fact two choices for $e$ ) and $\sigma$ (there can be two choices for $\sigma$ if the endpoint lies on a wedge boundary), so $v$ and $v^{\prime}$ are also determined (at worst there can be four such pairs). The total number of vertices $v$ in this subcase is thus $O\left(m_{2}\right)$. We may thus assume that $e$ fully crosses the wedge $W(\sigma)$.

Trace $\sigma \cap \partial a$ from $v$ and from $v^{\prime}$ into $P_{b}$. If we reach an endpoint $w$ of $\sigma \cap \partial a$ along one of these arcs (as in Fig. 7(a)), we charge $v$ and $v^{\prime}$ to $w$, note that, as above, $w$ can be charged at most twice, and conclude that the number of vertices $v$ in this subcase is $O\left(\lambda_{s}\left(m_{1}\right)\right)$. Otherwise, each of these arcs is crossed by another edge of the inner fat triangle $\Delta$ bounded by $e$ (see Figure 7(b)). The analysis in Lemma 3.4 implies that it is impossible that such an edge $e^{\prime} \neq e$ crosses both arcs, and it is impossible for such an $e^{\prime}$ to cross the same arc twice (in both cases one of the angles of $\Delta$ would have to be at most $\left.2 \pi / t<\beta_{0}\right)$. Since the common endpoint $z$ of $e$ and $e^{\prime}$ lies outside the wedge $W(\sigma)$, it follows that as we trace $e^{\prime}$ from $z$, we first meet the wedge boundary (still outside $\sigma$ ) and then cross $\sigma \cap \partial a$. Since this must also hold for the third edge $e^{\prime \prime}$ of $\Delta$, it follows that 


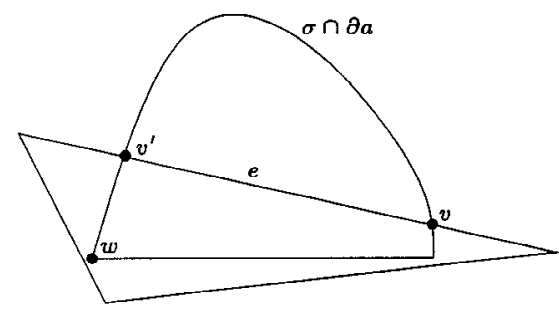

(a)

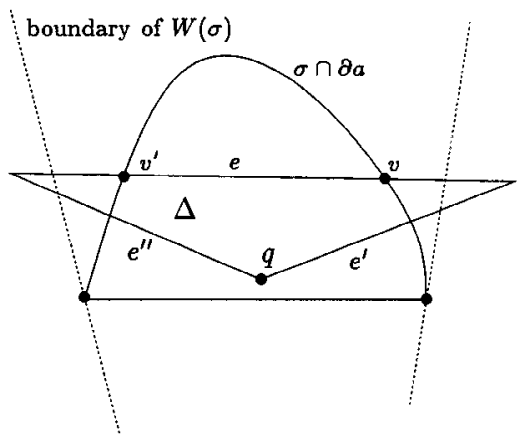

(b)

Fig. 7. Two cases where $P_{b}$ and the origin lie on the same side of the line containing $e$.

the vertex $q$ of $\Delta$ where $e^{\prime}$ and $e^{\prime \prime}$ meet must lie inside $\sigma$. We can thus charge $v$ and $v^{\prime}$ to $q$, note that the charging is unique (knowing $q$ we also know $\sigma$ and $\Delta$ ), and conclude that the number of vertices $v$ in this subcase is $O\left(m_{2}\right)$.

We have thus shown that the complexity of $U^{*}$ is $O\left(\left(\lambda_{s}\left(m_{1}\right)+m_{2}\right) \log \log n\right)$.

Step III. We finally turn to estimate the number of mixed vertices of the union $U$ of $\mathcal{C}_{1} \cup \mathcal{C}_{2}$

First, we take all the mixed vertices of $U^{*}$ and connect them to the origin, thereby splitting some of the slices of $U_{1}$ that were constructed at step II into subslices. The total number of new slices is $O\left(\left(\lambda_{s}\left(m_{1}\right)+m_{2}\right) \log \log n\right)$. Second, we concentrate only on irregular vertices of the union. We later exploit Theorem 2.1 to take into account regular vertices as well.

Let $v$ be an irregular vertex of $U$, lying on the boundary of a set $a \in \mathcal{C}_{1}$ and a set $b \in \mathcal{C}_{2}$. Let $\sigma$ be the (new) slice of $U_{1}$ containing $v$. With no loss of generality, we may assume that when we follow $\partial \sigma$ from $v$ in the counterclockwise direction, we enter into $b$. The complementary type of irregular vertices (where we enter $b$ as we go clockwise from $v$ along $\partial \sigma$ ) will be handled by a fully symmetric analysis.

We further classify these vertices into two subcategories: For each $b \in \mathcal{C}_{2}$, the two tangents from the origin to $b$ divide $\partial b$ into two arcs, one being "visible" from the origin and the other being "invisible." We refer to these portions as the lower boundary and upper boundary of $b$, respectively. We consider separately vertices $v$ as above that lie on the lower boundaries of the sets of $\mathcal{C}_{2}$ and vertices that lie on the upper boundaries.

Irregular Vertices on Lower Boundaries. Let $v$ and $v^{\prime}$ be two vertices as above, lying within a single new slice $\sigma$ and incident to the lower boundaries of two respective and distinct sets $b, b^{\prime} \in \mathcal{C}_{2}$, see Fig. 8. With no loss of generality, assume that $v$ lies clockwise to $v^{\prime}$. Consider the portion $\gamma$ of $\partial a$ between $v$ and $v^{\prime}$. The arc $\gamma$ partially overlaps the interior of $b$ near $v$ and it must cross $\partial b$ again. If it only crosses the lower boundary of $b$, then it is easily checked that $\partial a$ and $\partial b$ intersect only twice, so $v$ is a regular vertex, contrary to assumption. Hence, $\gamma$ also crosses the upper boundary of $b$. However, then, by construction, $\gamma$ must also cross the boundary of $P_{b}$, and thus it must contain a vertex 


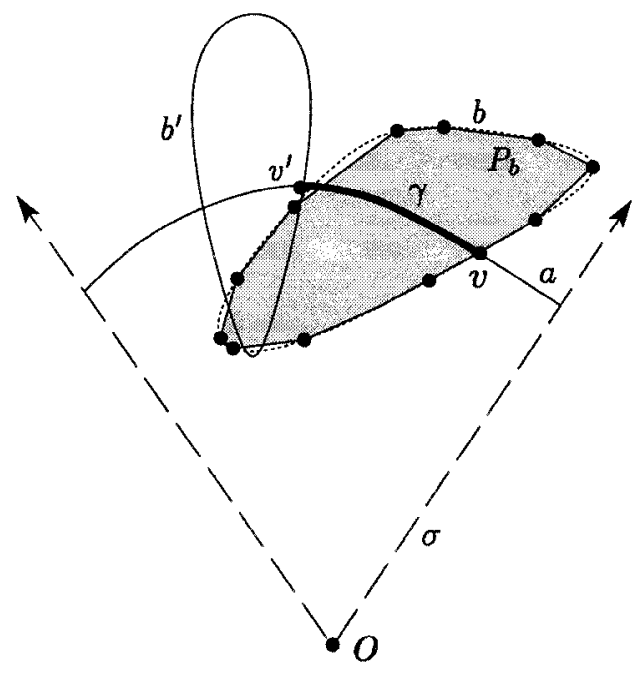

Fig. 8. The case of vertices on lower boundaries.

of $U^{*}$, which implies, by construction, that $v$ and $v^{\prime}$ cannot belong to the same new slice, again a contradiction.

We thus conclude that any new slice can have at most one vertex of the union of the type under consideration (i.e., a vertex that lies on the lower boundary of the set of $\mathcal{C}_{2}$ it is incident to and we enter this set as we follow $\partial \sigma$ counterclockwise from that vertex), so the number of these vertices is $O\left(\left(\lambda_{s}\left(m_{1}\right)+m_{2}\right) \log \log n\right)$. By repeating the analysis to the complementary type of lower-boundary vertices, we conclude that the overall number of vertices on lower boundaries is $O\left(\left(\lambda_{s}\left(m_{1}\right)+m_{2}\right) \log \log n\right)$.

Irregular Vertices on Upper Boundaries. We fix the new slice $\sigma$, as above, and consider the number $N_{\sigma}$ of sets in $\mathcal{C}_{2}$ whose upper boundaries are incident to vertices $v$ of the above kind that lie on $\sigma \cap \partial a$. If such a set $b$ has a supporting line that passes through the origin and is contained in the wedge $W(\sigma)$, then we charge the corresponding vertex or vertices to that line. This charging is almost unique, since the line determines both the set $b$ and the slice $\sigma$. Hence the number of vertices $v$ of this kind is at most $s m_{2}$. We can therefore exclude such pairs $(b, \sigma)$ from our analysis.

Let $v$ and $v^{\prime}$ be two vertices as above, lying within a single new slice $\sigma$, incident to the upper boundaries of two respective and distinct sets $b, b^{\prime} \in \mathcal{C}_{2}$, and have the property that we enter $b$ (resp. $b^{\prime}$ ) as we move from $v$ (resp. $v^{\prime}$ ) along $\partial \sigma$ counterclockwise; see Fig. 9. With no loss of generality, assume that $v$ lies clockwise to $v^{\prime}$. Consider the portion $\gamma$ of $\partial a$ (the set of $\mathcal{C}_{1}$ that bounds $\sigma$ ) between $v$ and $v^{\prime}$. As above, the $\operatorname{arc} \gamma$ partially overlaps the interior of $b$ near $v$ and it must cross $\partial b$ again. If it crosses the lower boundary of $b$, then, arguing as above, $\gamma$ must contain a vertex of $U^{*}$, which is impossible. Hence $\gamma$ only crosses the upper boundary of $b$. The same argument also implies that $\gamma$ cannot cross the lower boundary of $b^{\prime}$.

We claim that $\partial b$ and $\partial b^{\prime}$ must intersect within the wedge bounded by the two rays $\overrightarrow{o v}, \overrightarrow{o v^{\prime}}$, emerging from the origin toward $v$ and $v^{\prime}$, respectively. Indeed, if this were false, 


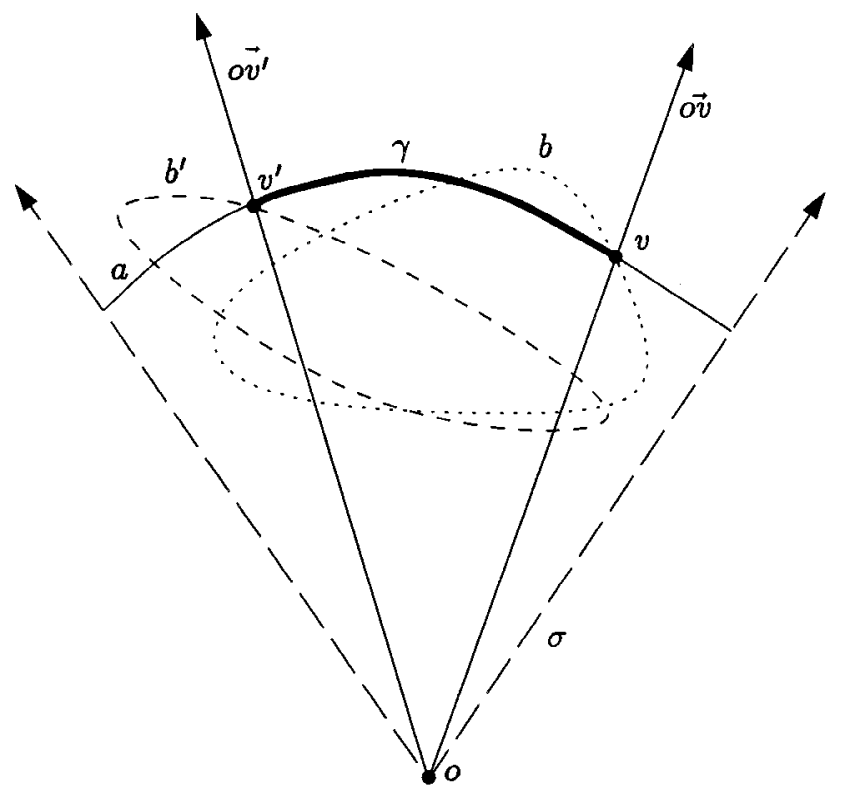

Fig. 9. $\partial b$ and $\partial b^{\prime}$ must cross inside the wedge bounded between the rays $\overrightarrow{o v}$ and $\overrightarrow{o v^{\prime}}$.

then either the ray $\overrightarrow{o v}$ would have to intersect $b^{\prime}$ in a segment disjoint from the segment $o v$, or the ray $\overrightarrow{s v^{\prime}}$ would have to intersect $b$ in a segment disjoint from $o v^{\prime}$ (in the present subcase, both rays must cross both objects). However, either of these configurations would imply that $\gamma$ crosses the lower boundary of either $b$ or $b^{\prime}$, which, as we have just argued, is impossible. This establishes our claim. See Fig. 9 for an illustration.

We choose some threshold parameter $k$, and consider the following two cases:

Case $(a): N_{\sigma} \leq k$. Since the boundary of each of these $N_{\sigma}$ sets intersects $\partial a$ in at most $s / 2$ vertices $v$ of the type considered here, it follows that $\sigma \cap \partial a$ contains at most $s k / 2$ such vertices. Summing this bound over all new slices $\sigma$ with $N_{\sigma} \leq k$, the overall number of vertices of $U$ of this type in these slices is at most

$$
O\left(k\left(\lambda_{s}\left(m_{1}\right)+m_{2}\right) \log \log n\right) .
$$

Case (b): $N_{\sigma}>k$. As argued above, the boundary of each of the $N_{\sigma}$ sets of $\mathcal{C}_{2}$ that are incident to the vertices under consideration intersects at least $k$ other such boundaries within the angular span of $W(\sigma)$. It follows that the arrangement $\mathcal{A}\left(\mathcal{C}_{2}\right)$ contains $\Omega\left(k N_{\sigma}\right)$ vertices at level at most $k$ (i.e., vertices contained in at most $k$ other sets of $\mathcal{C}_{2}$ ). On the other hand, arguing as in case (a), the number of vertices $v$ of the above kind that are incident to $\sigma \cap \partial a$ is $\leq s N_{\sigma} / 2$. Hence, the number of these vertices is at most $O\left(N_{\sigma} /\left(k N_{\sigma}\right)\right)=O(1 / k)$ times the number of vertices of $\mathcal{A}\left(\mathcal{C}_{2}\right)$ within $W(\sigma)$ at level at most $k$. Summing this inequality over all relevant slices $\sigma$, the overall number of such vertices is

$$
O\left(\frac{1}{k} F_{\leq k}\left(\mathcal{C}_{2}\right)\right)
$$


where $F_{\leq k}\left(\mathcal{C}_{2}\right)$ is the number of vertices of $\mathcal{A}\left(C_{2}\right)$ at level at most $k$. Using the ClarksonShor probabilistic analysis technique [6], we have $F_{\leq k}\left(\mathcal{C}_{2}\right)=O\left(k^{2} F\left(m_{2} / k\right)\right)$. By the induction hypothesis, we have

$$
\begin{aligned}
F\left(\frac{m_{2}}{k}\right) & \leq\left(\frac{m_{2}}{k}\right)^{1+c \sqrt{\log \log \left(m_{2} / k\right) / \log \left(m_{2} / k\right)}}=\frac{m_{2}}{k} \cdot 2^{c \sqrt{\log \log \left(m_{2} / k\right) \log \left(m_{2} / k\right)}} \\
& \leq \frac{m_{2}}{k} \cdot 2^{c \sqrt{\log \left(m_{2} / k\right) \log \log n}}
\end{aligned}
$$

Hence, collecting all bounds, and taking into account regular vertices too (using Theorem 2.1), we obtain that the number of mixed vertices of $U$ is at most

$$
C\left(k\left(\lambda_{s}\left(m_{1}\right)+m_{2}\right) \log \log n+m_{2} \cdot 2^{c \sqrt{\log \left(m_{2} / k\right) \log \log n}}\right),
$$

for some constant $C$ that depends on $\alpha$ and $s$.

As outlined above, we now sum this bound over all nodes $\xi$ and $\eta$ of our segment tree and over the constant number of "stabbing points" (the points of $P_{\eta}$ ) used in each secondary node $\eta$, observing that the sums of the quantities $m_{1}, m_{2}$ are both $O\left(n \log ^{2} n\right)$. Hence the total number of vertices of the union is

$$
C\left(k \lambda_{s}(n) \log ^{2} n \log \log n+n \log ^{2} n \cdot 2^{c \sqrt{\log (n / k) \log \log n}}\right) .
$$

Using the inequality

$$
\sqrt{\log n}-\sqrt{\log (n / k)}=\frac{\log k}{\sqrt{\log n}+\sqrt{\log (n / k)}} \geq \frac{\log k}{2 \sqrt{\log n}},
$$

we obtain

$$
\begin{gathered}
C n \log ^{2} n \cdot 2^{c \sqrt{\log (n / k) \log \log n}} \leq \frac{C n \log ^{2} n \cdot 2^{c \sqrt{\log n \log \log n}}}{2^{(c / 2) \log k \sqrt{\log \log n / \log n}}} \\
\leq n \cdot 2^{c \sqrt{\log n \log \log n}} \cdot \frac{C \log ^{2} n}{k^{(c / 2) \sqrt{\log \log n / \log n}}} .
\end{gathered}
$$

We now choose $k=2^{c^{\prime}} \sqrt{\log n \log \log n}$, for some constant $c^{\prime}<c$, and observe that the above bound becomes

$$
n \cdot 2^{c \sqrt{\log n \log \log n}} \cdot C \log ^{2-c c^{\prime} / 2} n \ll n \cdot 2^{c \sqrt{\log n \log \log n}},
$$

provided that $c$ and $c^{\prime}$ are sufficiently large. The left term of (2) is also seen to be much smaller than $n \cdot 2^{c \sqrt{\log n \log \log n}}$, assuming $c>c^{\prime}$ and $n$ is sufficiently large. Hence, with an appropriate choice of $n_{0}, c$, and $c^{\prime}$, the induction assertion also holds for $F(n)$. This concludes the proof of Theorem 1.1. 


\section{Conclusions}

In this paper we have established a near-linear upper bound on the complexity of the union of $n$ compact convex $\alpha$-fat objects so that each pair of object boundaries intersect in at most some constant number of points.

This result raises several open problems:

- The first problem is to reduce the upper bound to a bound of the form $O$ ( $n$ polylog $(n))$. A significant step toward this goal has recently been accomplished (after the original submission of this paper) by Efrat [7] and by Efrat and Katz [8], with the only reservation that the sets that they consider must essentially have bounded curvature.

- Another problem is to extend our result to nonconvex fat regions. Here the notion of fatness is less clearly defined; see [21] for several possible definitions. The previously cited papers [7], [8] obtain partial results in this direction.

- Finally, the challenge is to extend this result to three and higher dimensions. In 3 -space the goal is to obtain near-quadratic bounds on the complexity of the union of fat convex objects of various kinds. Most instances of this problem are wide open. Even the case of the union of (arbitrarily aligned) cubes is not known.

\section{Acknowledgment}

We wish to thank János Pach for helpful discussions concerning this problem. These discussions have led to the paper [19], on whose main result (Theorem 2.1) the current paper strongly depends.

\section{References}

1. P. K. Agarwal, E. F. Grove, T. M. Murali and J. S. Vitter, Binary space partitions for fat rectangles, Proc. 37th Ann. IEEE Symp. on Foundations of Computer Science, 1996, pp. 482-491.

2. P. Agarwal, M. Katz and M. Sharir, Computing depth order and related problems, Comput. Geom. Theory Appl. 5 (1995), 187-206.

3. H. Alt, R. Fleischer, M. Kaufmann, K. Mehlhorn, S. Näher, S. Schirra and C. Uhrig, Approximate motion planning and the complexity of the boundary of the union of simple geometric figures, Algorithmica 8 (1992), 391-406.

4. M. de Berg, Linear size binary space partitions for fat objects, Proc. 3rd Ann. European Symp. on Algorithms, Lecture Notes in Computer Science, vol. 979, Springer-Verlag, Berlin, 1995, pp. 252-263.

5. B. Chazelle, H. Edelsbrunner, L. Guibas and M. Sharir, Algorithms for bichromatic line segment problems and polyhedral terrains, Algorithmica 11 (1994), 116-132.

6. K. Clarkson and P. Shor, Applications of random sampling in computational geometry, II, Discrete Comput. Geom. 4 (1989), 387-421.

7. A. Efrat, The complexity of the union of $(\alpha, \beta)$-covered objects, Proc. 15th ACM Symp. on Computational Geometry, 1999, pp. 134-142.

8. A. Efrat and M. Katz, On the union of $\alpha$-curved objects, Proc. 14th ACM Symp. on Computational Geometry, 1998, pp. 206-213.

9. A. Efrat, M. J. Katz, F. Nielsen and M. Sharir, Dynamic data structures for fat objects and their applications, Proc. 5th Workshop on Algorithms and Data Structures, 1997, pp. 297-306. 
10. A. Efrat, G. Rote and M. Sharir, On the union of fat wedges and separating a collection of segments by a line, Comput. Geom. Theory Appl. 3 (1993), 277-288.

11. A. Efrat and M. Sharir, The complexity of the union of fat objects in the plane, Proc. 13th ACM Symp. on Computational Geometry, 1997, pp. 104-112.

12. A. Efrat, M. Sharir and A. Ziv, Computing the smallest $k$-enclosing circle and related problems, Comput. Geom. Theory Appl. 4 (1994), 119-136.

13. M. Katz, 3-D vertical ray shooting and 2-D point enclosure, range searching, and arc shooting amidst convex fat objects, Comput. Geom. Theory Appl. 8 (1997), 299-316. Also in Tech. Report 2583, INRIA, Sophia-Antipolis, 1995.

14. M. Katz, M. Overmars and M. Sharir, Efficient output sensitive hidden surface removal for objects with small union size, Comput. Geom. Theory Appl. 2 (1992), 223-234.

15. K. Kedem, R. Livne, J. Pach and M. Sharir, On the union of Jordan regions and collision-free translational motion amidst polygonal obstacles, Discrete Comput. Geom. 1 (1986), 59-71.

16. J. Matoušek, J. Pach, M. Sharir, S. Sifrony and E. Welzl, Fat triangles determine linearly many holes, SIAM J. Comput. 23 (1994), 154-169.

17. M. H. Overmars, Point location in fat subdivisions, Inform. Process. Lett. 44 (1992), 261-265.

18. M. H. Overmars and A. F. van der Stappen, Range searching and point location among fat objects, J. Algorithms 21 (1996), 629-656.

19. J. Pach and M. Sharir, On the boundary of the union of planar convex sets, Discrete Comput. Geom. 21 (1999), 321-328.

20. M. Sharir and P. K. Agarwal, Davenport-Schinzel Sequences and Their Geometric Applications, Cambridge University Press, New York, 1995.

21. F. van der Stappen, Motion Planning amidst Fat Obstacles, Ph.D Dissertation, Utrecht University, 1994.

22. A. F. van der Stappen, D. Halperin and M. H. Overmars, The complexity of the free space for a robot moving amidst fat obstacles, Comput. Geom. Theory Appl. 3 (1993), 353-373.

23. M. van Kreveld, On fat partitioning, fat covering, and the union size of polygons, Comput. Geom. Theory Appl. 9 (1998), 452-463.

Received April 7, 1998, and in revised form August 24, 1999. 